\title{
Boundary-layer flow of a micropolar fluid due to a stretching wall
}

\author{
T. -Y. Na, I. Pop
}

Summary A Theoretical analysis is carried out to study the boundary-layer flow over a continuously moving surface through an otherwise quiescent micropolar fluid. The transformed boundary-layer equations are solved numerically for a power-law surface velocity using the Keller-box method. The effects of the micropolar $\mathrm{K}$ and exponent $\mathrm{m}$ parameters on the velocity and microrotation field as well as on the skin-friction group are discussed in a detailed manner. It is shown that there is a near-similarity solution of this problem. The accuracy of the present solution is also discussed.

Key words polymer, extrusion, boundary layer, micropolar fluid, similarity

1

Introduction

Polymer sheets and filaments are manufactured by continuous extrusion of the polymer from a die to a windup roller which is located at a finite distance away. In the manufacture of the polymer sheets, the material is in a molten phase when thrust through an extrusion die, and then cools and solidifies some distance away from the die before arriving at the collecting stage. The thin polymer sheet constitutes a continously moving solid surface with a non-uniform surface velocity through an otherwise quiescent fluid. Due to the entrainment of ambient fluid this boundary layer flow is quite different from that over a semi-infinite flat plate. Sakiadis [1] was probably the first to recognize this new class of problems. Lately, this problem has been considered by many investigators including [2], [3], [4], [5], and [6] among others.

The inadequacy of the classical Navier-Stokes theory to describe rheologically complex fluids such as liquid crystals, animal blood, etc., has led to the development of microcontinuum fluid mechanics as an extension of the classical theory. Many models have been proposed to take into account the mechanically significant microstructure of such fluids, see [7]. One of the models, the theory of micropolar fluids introduced in $[8,9]$, has generated considerable interest in the literature. In this theory, the micropolar fluid exhibits the microrotation effects and microrotational inertia. Such fluids can support couple stress and body couples only. Physically they may represent fluids with bar-like or sphere-like elements.

The present paper aims to study the laminar boundary-layer flow of a micropolar fluid over a continously moving surface. We consider those micropolar fluids that give rise to a sheet speed which is proportional to $x^{m},[10],[14]$ and [15], and will, therefore, not be presented here. Results are given for different values of the parameter sets $K$ and $\xi^{*}$ with $m=0,0.5$ and 1.0 .

Typical velocity and microrotation profiles are displayed in Figs. 2 and 3 for $K=0,1$ and 2 with $m=0.5$. The profiles appear the same for all $\xi^{*}$. For this reason, Fig. 4 gives the plots of SFG for these values of $K$ and $m$ which again is the same for all $\xi^{*}$. These figures show that the

Accepted for publication 1 April 1996

T. -Y. Na

Department of Mechanical Engineering, University of Michigan Dearborn, Dearborn, MI, 48128, USA

I. Pop

Faculty of Mathematics, University of Cluj, R-3400 Cluj, CP253, Romania 


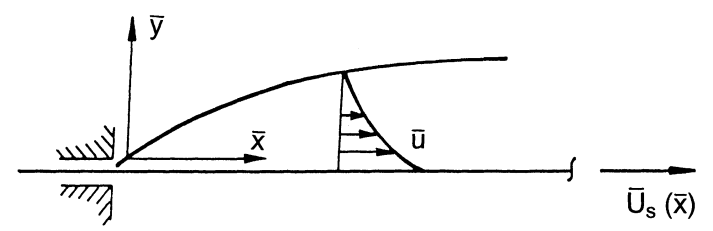

Fig. 1. Physical model and coordinate system

velocity profiles increase with the increase of the micropolar parameter $K$, while the microrotation profiles first decrease and then increase as $K$ increases. Further, it is seen from Fig. 4 that the skin friction increases as both the parameters $m$ and $K$ increase. We also notice that the present results are in excellent agreement with those reported in [16] for the corresponding viscous $(K=0)$ problem.

The similarity nature of the solutions presented in Figs. 2 through 4 deserves further investigation. Tables 1 and 2 demonstrate the variation of SFG with $\xi^{*}$ for the non-similar case and the similar case respectively. In Table 1, the change is so small that it will not be able to show in graphic form. In the similarity case, however, the values of SFG are indeed identical for all $\xi^{*}$. The first case will now be designated as near-similarity solution, since it is non-similar but the variation is so small that for the practical range of the physical parameters the change is only in the sixth digit.

This near-similarity behavior can also be observed by direct examination of Eqs. (30) and (31). It is straightforward to check that setting $2 g+f^{\prime \prime}=0$ where $x$ is the non-dimensional distance measured along the sheet surface. The constant of proportionality and the exponent $m$ can both be regarded as materially dependent constants. The quality of the resulting sheet material, as well as the cost of production, is affected by the speed of collection and the heat transfer rate, and a knowledge of the flow properties of the ambient micropolar fluid is clearly desirable. In what follows, we shall assume that the flow is steady and such that the boundarylayer equations are appropriate. We consider the velocity and microrotation fields only. The governing equations are first transformed for easier solution, and then solved numerically using a very efficient finite-difference scheme known as Keller-box method developed in [10]. The effects of the non-dimensional micropolar parameter $K$ and exponent $m$ on the velocity and microrotation fields as well as on the skin friction group are discussed.

\section{2}

\section{Basic equations}

Consider the flow of a steady, laminar, incompressible micropolar fluid past an extensible sheet which is moving continously in the positive $\bar{x}$ - direction with an arbitrary surface velocity $\bar{U}_{S}(\bar{x})$. The orthogonal coordinates $\bar{x}, \bar{y}$ are measured along the sheet and, respectively, normal to it with the origin at a fixed point $O$ (die slot). Figure 1 shows the flow model and coordinate system. The full equations governing the two-dimensional flow of a micropolar fluid of a micropolar fluid are, see [11],

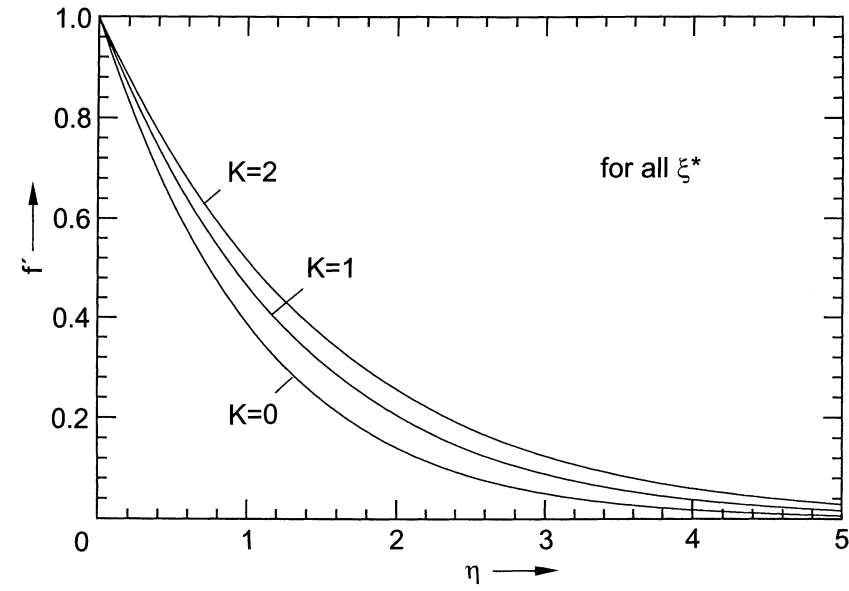

Fig. 2. Velocity profiles $f^{\prime}$ for $m=0.5$ and $K=0,1$ and 2 

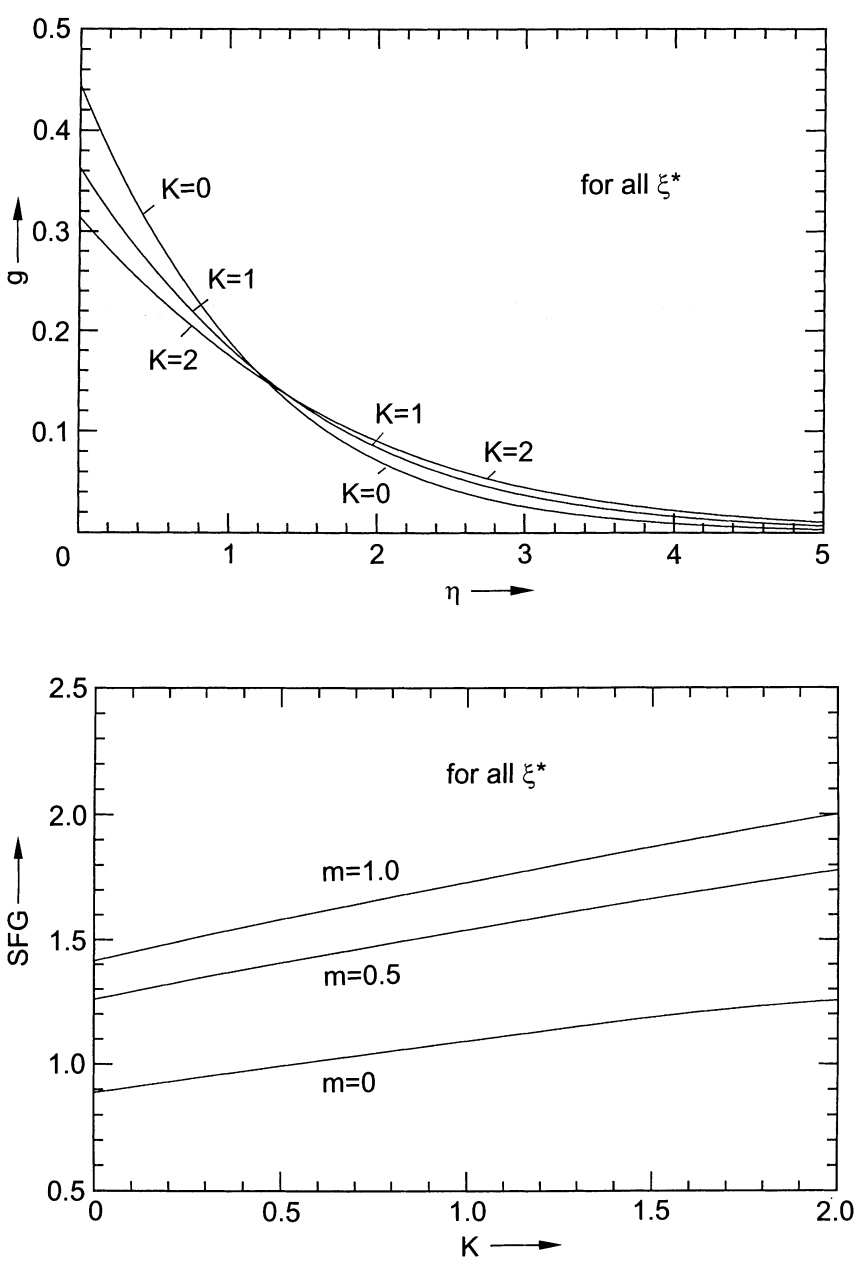

Fig. 3. Microrotation profiles $g$ for $m=0.5$ and $K=0,1$ and 2
Fig. 4. Skin friction group SFG for $m=0,0.5$ and 1.0

$$
\begin{aligned}
& \frac{\partial \bar{u}}{\partial \bar{x}}+\frac{\partial \bar{v}}{\partial \bar{y}}=0 \\
& \rho\left(\bar{u} \frac{\partial \bar{u}}{\partial \bar{x}}+\bar{v} \frac{\partial \bar{u}}{\partial \bar{y}}\right)=-\frac{\partial \bar{p}}{\partial \bar{x}}+(\mu+\kappa)\left(\frac{\partial^{2} \bar{u}}{\partial \bar{x}^{2}}+\frac{\partial^{2} \bar{u}}{\partial \bar{y}^{2}}\right)+\kappa \frac{\partial \bar{N}}{\partial \bar{y}},
\end{aligned}
$$

Table 1. Near-similar solutions $(K=2, m=0.5)$

\begin{tabular}{ll}
\hline$\xi^{*}$ & SFG \\
\hline 0.0000000000 & 1.7793572861 \\
0.0000100000 & 1.7793573653 \\
0.0000300000 & 1.7793574202 \\
0.0000700000 & 1.7793574362 \\
0.0001500000 & 1.7793574585 \\
0.0003100000 & 1.7793574879 \\
0.0006300000 & 1.7793575120 \\
0.0012700000 & 1.7793575389 \\
0.0025499999 & 1.7793575644 \\
0.0051099999 & 1.7793575889 \\
0.0102299997 & 1.7793576124 \\
0.0204699995 & 1.7793576335 \\
0.0409499990 & 1.7793576531 \\
0.0819099979 & 1.7793576701 \\
0.1638299959 & 1.7793576854 \\
0.3276699917 & 1.7793576986 \\
0.6553499834 & 1.7793577104 \\
1.3107099669 & 1.7793577201 \\
2.6214299338 & 1.7793577248 \\
5.2428698676 & 1.7793577222 \\
\hline
\end{tabular}


Table 2. Similar solutions $(K=2, m=1.0)$

\begin{tabular}{ll}
\hline$\xi^{*}$ & SFG \\
\hline 0.0000000000 & 2.0003020199 \\
0.0000100000 & 2.0003020199 \\
0.0000300000 & 2.0003020199 \\
0.0000700000 & 2.0003020199 \\
0.0001500000 & 2.0003020199 \\
0.0003100000 & 2.0003020199 \\
0.0006300000 & 2.0003020199 \\
0.0012700000 & 2.0003020199 \\
0.0025499999 & 2.0003020199 \\
0.0051099999 & 2.0003020199 \\
0.0102299997 & 2.0003020199 \\
0.0204699995 & 2.0003020199 \\
0.0409499990 & 2.0003020199 \\
0.0819099979 & 2.0003020199 \\
0.1638299959 & 2.0003020199 \\
0.3276699917 & 2.0003020199 \\
0.6553499834 & 2.0003020199 \\
1.3107099669 & 2.0003020199 \\
2.6214299338 & 2.0003020199 \\
5.2428698676 & 2.0003020199 \\
\hline
\end{tabular}

$\rho\left(\bar{u} \frac{\partial \bar{v}}{\partial \bar{x}}+\bar{v} \frac{\partial \bar{v}}{\partial \bar{y}}\right)=-\frac{\partial \bar{p}}{\partial \bar{y}}+(\mu+\kappa)\left(\frac{\partial^{2} \bar{v}}{\partial \bar{x}^{2}}+\frac{\partial^{2} \bar{v}}{\partial \bar{y}^{2}}\right)-\kappa \frac{\partial \bar{N}}{\partial \bar{x}}$,

$\rho j\left(\bar{u} \frac{\partial \bar{N}}{\partial \bar{x}}+\bar{v} \frac{\partial \bar{N}}{\partial \bar{y}}\right)=-2 \kappa \bar{N}+\kappa\left(\frac{\partial \bar{v}}{\partial \bar{x}}-\frac{\partial \bar{u}}{\partial \bar{y}}\right)+\operatorname{div}(\gamma \nabla \bar{N})$

where $(\bar{u}, \bar{v})$ are the velocity components along $(\bar{x}, \bar{y})$-axes, $\bar{p}$ is the pressure, $\bar{N}$ is the component of the microrotation whose direction of rotation is in the $(\bar{x}, \bar{y})$ plane and $j$ is the microinertia density. Further, $\rho$ is the fluid density, $\mu$ is the dynamic viscosity, $\kappa$ is the microrotation parameter and $\gamma$ is the spin-gradient viscosity given by, see [11],

$\gamma=\left(\mu+\frac{\kappa}{2}\right) j$.

We follow the work of many recent authors by assuming that $j$ is a constant, and therefore it shall be set equal to a reference value, $j_{0}$.

The appropriate boundary conditions of Eqs. (1) to (4) are

$\bar{v}=0, \bar{u}=\bar{U}_{s}(\bar{x}), \bar{N}=-n \frac{\partial \bar{u}}{\partial \bar{y}} \quad$ on $\quad y=0$,

$\bar{v} \rightarrow 0, \bar{u} \rightarrow 0, \bar{N} \rightarrow 0 \quad$ as $\quad \bar{y} \rightarrow \infty$

In Eq. (5) we have followed [12] in assigning a variable relation between $\bar{N}$ and the skin friction at the surface. The value of $n=0$ represents cases where the particle density is sufficiently great that the microelements close to the wall are unable to rotate. The value $n=1 / 2$ corresponds to weak concentrations, and when $n=1$ we have cases which are representative of turbulent boundary layer. We shall consider here only the value of $n$ equal to $1 / 2$.

By employing the following non-dimensionalization

$\hat{x}=\frac{\bar{x}}{\ell}, \hat{y}=\frac{\bar{y}}{\ell}, \hat{u}=\frac{\bar{u}}{U_{0}}, \hat{v}=\frac{\bar{v}}{U_{0}}, \hat{p}=\frac{\bar{p}}{\rho U_{0}^{2}}, \hat{N}=\frac{\bar{N}}{U_{0} / \ell}, \hat{U}_{s}(\hat{x})=\frac{\bar{U}_{s}(\bar{x})}{U_{0}}$

where $U_{0}$ is a characteristic velocity and $\ell$ is a characteristic length defined as $j_{0}=\ell^{2}$, we arrive at

$\frac{\partial \hat{u}}{\partial \hat{x}}+\frac{\partial \hat{v}}{\partial \hat{y}}=0$ 
$\hat{u} \frac{\partial \hat{u}}{\partial \hat{x}}+\hat{v} \frac{\partial \hat{u}}{\partial \hat{y}}=-\frac{\partial \hat{p}}{\partial \hat{x}}+\left(\frac{1+K}{R e}\right)\left(\frac{\partial^{2} \hat{u}}{\partial \hat{x}^{2}}+\frac{\partial^{2} \hat{u}}{\partial \hat{y}^{2}}\right)+\frac{K}{R e} \frac{\partial \hat{N}}{\partial \hat{y}}$

$\hat{u} \frac{\partial \hat{v}}{\partial \hat{x}}+\hat{v} \frac{\partial \hat{v}}{\partial \hat{y}}=-\frac{\partial \hat{p}}{\partial \hat{y}}+\left(\frac{1+K}{R e}\right)\left(\frac{\partial^{2} \hat{v}}{\partial \hat{x}^{2}}+\frac{\partial^{2} \hat{v}}{\partial \hat{y}^{2}}\right)-\frac{K}{R e} \frac{\partial \hat{N}}{\partial \hat{x}}$,

$\hat{u} \frac{\partial \hat{N}}{\partial \hat{x}}+\hat{v} \frac{\partial \hat{N}}{\partial \hat{y}}=-2 \frac{K}{R e}\left(\frac{\partial \hat{v}}{\partial \hat{x}}-\frac{\partial \hat{u}}{\partial \hat{y}}\right)+\left(\frac{1+K / 2}{R e}\right)\left(\frac{\partial^{2} \hat{N}}{\partial \hat{x}^{2}}+\frac{\partial^{2} \hat{N}}{\partial \hat{y}^{2}}\right)$,

subject to the boundary conditions

$\hat{v}=0, \hat{u}=\hat{U}_{s}(\hat{x}), \hat{N}=-\frac{1}{2} \frac{\partial \hat{u}}{\partial \hat{y}} \quad$ on $\quad \hat{y}=0$

$\hat{u} \rightarrow 0, \hat{v} \rightarrow 0, \hat{N} \rightarrow 0 \quad$ as $\quad \hat{y} \rightarrow \infty$

Here, $K=\kappa / \mu$, and $R e=U_{0} \ell / v$ is the Reynolds number.

We now invoke the boundary layer approximation by formally letting $R e$ very large, i.e. $R e \rightarrow \infty$, and setting

$\hat{x}=\operatorname{Re} x, \hat{y}=y, \hat{u}=\frac{\partial \psi}{\partial y}, \hat{v}=-R^{-1} \frac{\partial \psi}{\partial x}$,

where $\psi$ is the stream function. Equations (9) to (12) become

$\frac{\partial \psi}{\partial y} \frac{\partial^{2} \psi}{\partial x \partial y}-\frac{\partial \psi}{\partial x} \frac{\partial^{2} \psi}{\partial y^{2}}=(1+K) \frac{\partial^{3} \psi}{\partial y^{3}}+K \frac{\partial N}{\partial y}$,

$\frac{\partial \psi}{\partial y} \frac{\partial N}{\partial x}-\frac{\partial \psi}{\partial x} \frac{\partial N}{\partial y}=-K\left(2 N+\frac{\partial^{2} \psi}{\partial y^{2}}\right)+\left(1+\frac{K}{2}\right) \frac{\partial^{2} N}{\partial y^{2}}$

subject to the boundary conditions

$\psi=0, \frac{\partial \psi}{\partial y}=U_{s}(x), N=-\frac{1}{2} \frac{\partial^{2} \psi}{\partial y^{2}} \quad$ on $\quad y=0$,

$\frac{\partial \psi}{\partial y} \rightarrow 0, N \rightarrow 0 \quad$ as $\quad y \rightarrow \infty$

The original $x, y$ coordinates are now transformed by

$\xi=\int_{0}^{x} U_{s}(x) d x, \eta=(2 \xi)^{-\frac{1}{2}} U_{s} y$.

Also, the stream function $\psi$ and the microrotation component $N$ are introduced as

$\psi(x, y)=(2 \xi)^{\frac{1}{2}} f(\xi, \eta), N(x, y)=(2 \xi)^{-\frac{1}{2}} U_{s}^{2} g(\xi, \eta)$.

Substituting (20) and (21) into Eqs. (16) and (17), the functions $f$ and $g$ satisfy the following transformed equations :

$$
\begin{aligned}
& (1+K) f^{\prime \prime \prime}+f f^{\prime \prime}-\beta\left(f^{\prime}\right)^{2}+K g^{\prime}=2 \xi\left\{f^{\prime} \frac{\partial f^{\prime}}{\partial \xi}-f^{\prime \prime} \frac{\partial f}{\partial \xi}\right\} \\
& \left(1+\frac{1}{2} K\right) g^{\prime \prime}+f g^{\prime}+(1-2 \beta) f^{\prime} g-K \frac{2 \xi}{U_{s}^{2}}\left(f^{\prime \prime}+2 g\right)=2 \xi\left\{f^{\prime} \frac{\partial g}{\partial \xi}-g^{\prime} \frac{\partial f}{\partial \xi}\right\}
\end{aligned}
$$


which are subject to the boundary conditions

$f(\xi, 0)=0, f^{\prime}(\xi, 0)=1, g(\xi, 0)=-\frac{1}{2} f^{\prime \prime}(\xi, 0) ; f^{\prime}(\xi, \infty)=0, g(\xi, \infty)=0$.

Here, primes denote differentiation with respect to $\eta$ and $\beta$ is called a surface velocity parameter and is defined by

$\beta=\frac{2 \xi}{U_{s}} \frac{\mathrm{d} U_{s}}{\mathrm{~d} \xi}$

The skin friction at the wall $\bar{\tau}_{w}$ can be related to the non-dimensional wall skin-friction coefficient $C_{f}$ according to

$C_{f}=\frac{-2 \bar{\tau}_{w}}{\rho \bar{U}_{0}^{2}}$

where

$\bar{\tau}_{w}=\left[\mu\left(\frac{\partial \bar{u}}{\partial \bar{y}}+\frac{\partial \bar{v}}{\partial \bar{x}}\right)+K\left(\frac{\partial \bar{u}}{\partial \bar{y}}+\bar{N}\right)\right]_{\bar{y}=0}$.

Using (8), (15) and (21), the local skin-friction group can be formed as

$S F G=C_{f}\left(\frac{R e}{U_{s}^{2}}\right) \xi^{1 / 2}=-\frac{1}{\sqrt{2}}(2+K) f^{\prime \prime}(\xi, 0)$.

We consider now a surface velocity of the form

$U_{s}(x)=x^{m}$.

Equations (22) and (23) then become

$$
\begin{aligned}
& (1+K) f^{\prime \prime \prime}+f f^{\prime \prime}-\frac{2 m}{m+1}\left(f^{\prime}\right)^{2}+K g^{\prime}=2 \xi\left\{f^{\prime} \frac{\partial f^{\prime}}{\partial \xi}-f^{\prime \prime} \frac{\partial f}{\partial \xi}\right\} \\
& \left(1+\frac{1}{2} K\right) g^{\prime \prime}+f g^{\prime}+\frac{1-3 m}{1+m} f^{\prime} g-\frac{2 K \xi^{\frac{1-m}{1+m}}}{(m+1)^{\frac{2 m}{m+1}}}\left(f^{\prime \prime}+2 g\right)=2 \xi\left\{f^{\prime} \frac{\partial g}{\partial \xi}-g^{\prime} \frac{\partial f}{\partial \xi}\right\},
\end{aligned}
$$

subject to the boundary conditions given by (24).

It is worth mentioning that for $m=1$, Eqs. (30) and (31) become similar, i.e.

$$
\begin{aligned}
& (1+K) f^{\prime \prime \prime}+f f^{\prime \prime}-\left(f^{\prime}\right)^{2}+K g^{\prime}=0, \\
& \left(1+\frac{1}{2} K\right) g^{\prime \prime}+f g^{\prime}-f^{\prime} g-K\left(f^{\prime \prime}+2 g\right)=0,
\end{aligned}
$$

and are subject to the boundary conditions

$f(0)=0, f^{\prime}(0)=1, g(0)=-\frac{1}{2} f^{\prime \prime}(0) ; f^{\prime}(\infty)=0, g(\infty)=0$.

These equations describe the flow due to a stretching sheet which issues from a die slot into a micropolar fluid at rest. The second case for which Eqs. (30) and (31) possess similarity solutions arises when $K=0$ (Newtonian fluid). Thus, we have

$f^{\prime \prime \prime}+f f^{\prime \prime}-\frac{2 m}{m+1}\left(f^{\prime}\right)^{2}=0$ 
$g^{\prime \prime}+f g^{\prime}+\frac{1-3 m}{m+1} f^{\prime} g=0$

subject to the same boundary conditions (34). In this case, the flow field is unaffected by the microstructure of the fluid, and hence the component of the microrotation is a passive quantity. We notice in passing that Eq. (35) describes the boundary-layer flow over a continous moving flat plate in a quiescent viscous fluid, as described in [13].

On the other hand, it would appear from Eq. (31) that for $m<1$ the fourth term on the lefthand side would become increasingly dominant as $\xi \rightarrow \infty$. This, in turn, would indicate that $f^{\prime \prime}+2 g \rightarrow 0$, i.e., $g \rightarrow-(1 / 2) f^{\prime \prime}$ as $\xi \rightarrow \infty$. Hence, in order to observe the behaviour of the solution for large $\xi$, it was decided to compress the range of $\xi$ into a correspondingly smaller streamwise distance by introducing the variable $\xi^{*}$ defined as

$\xi^{*}=\frac{1}{2} \ln (\xi+1)$.

Equations (30) and (31) now become

$$
\begin{gathered}
(1+K) f^{\prime \prime \prime}+f f^{\prime \prime}-\frac{2 m}{m+1}\left(f^{\prime}\right)^{2}+K g^{\prime}=\left(1-e^{-2 \xi^{*}}\right)\left\{f^{\prime} \frac{\partial f^{\prime}}{\partial \xi^{*}}-f^{\prime \prime} \frac{\partial f}{\partial \xi^{*}}\right\} \\
\left(1+\frac{1}{2} K\right) g^{\prime \prime}+f g^{\prime}+\frac{1-3 m}{m+1} f^{\prime} g-\frac{2 k\left(e^{2 \xi^{*}}-1\right)^{\frac{1-m}{1+m}}}{(m+1)^{\frac{2 m}{m+1}}}\left(f^{\prime \prime}+2 g\right) \\
=\left(1-e^{-2 \xi^{*}}\right)\left\{f^{\prime} \frac{\partial g}{\partial \xi^{*}}-g^{\prime} \frac{\partial f}{\partial \xi^{*}}\right\}
\end{gathered}
$$

subject to the boundary conditions

$f\left(\xi^{*}, 0\right)=0, f^{\prime}\left(\xi^{*}, 0\right)=1, g\left(\xi^{*}, 0\right)=-\frac{1}{2} f^{\prime \prime}\left(\xi^{*}, 0\right)$,

$f^{\prime}\left(\xi^{*}, \infty\right)=0, g\left(\xi^{*}, \infty\right)=0$.

Also, the local skin-friction group is now given by

$S F G=-\frac{1}{\sqrt{2}}(2+K) f^{\prime \prime}\left(\xi^{*} .0\right)$.

\section{3}

Results and discussion

The system of partial differential equations (38) and (39) subject to the boundary conditions (40) and (41) were solved numerically using the Keller-box method. The details are essentially the same as those described in Eqs. (30) and (31) when $m<1$ yields the following consistent pair of similarity equations

$\left(1+\frac{1}{2} K\right) f^{\prime \prime \prime}+f f^{\prime \prime}-\frac{2 m}{m+1}\left(f^{\prime}\right)^{2}=0, g=-\frac{1}{2} f^{\prime \prime}$.

The similarity solution of these equations can be easily written in terms of the standard [13] boundary-layer equations by rescaling the similarity variables. Thus, if we take

$f(\eta)=\left(1+\frac{1}{2} K\right)^{1 / 2} \tilde{f}(\tilde{\eta}), g(\eta)=\left(1+\frac{1}{2} K\right)^{-1 / 2} \tilde{g}(\tilde{\eta}), \eta=\left(1+\frac{1}{2} K\right)^{1 / 2} \tilde{\eta}$,

we obtain

$\tilde{f}^{\prime \prime \prime}+\tilde{f} \tilde{f}^{\prime \prime}-\frac{2 m}{m+1}\left(\tilde{f}^{\prime}\right)^{2}=0, \tilde{g}=-\frac{1}{2} \tilde{f}^{\prime \prime}$ 
subject to the boundary conditions

$\tilde{f}(0)=0, \tilde{f}^{\prime}(0)=1, \tilde{f}^{\prime}(\infty)=0$.

Primes now denote differentiation with respect to $\tilde{\eta}$.

\section{References}

1. Sakiadis, B. C.: Boundary-layer behavior on continuous solid surfaces: I. The boundary-layer equations for two-dimensional and axisymmetric flow. Chemical Engineering Progress 7(1991)26-28

2. Tsou, F. K.; Sparrow, E. M.; Goldstein, R. J.: Flow and heat transfer in the boundary-layer on a continuous moving surface. Int. J.Heat Mass Transfer 10 (1967) 219-235

3. Jeng, D. R.; Chang, T. C. A.; De Witt, K. J.: Momentum and heat transfer on a continuous moving surface. Trans. ASME J. Heat Transfer 108 (1986) 532-539

4. Karwe, M. V.; Jaluria, Y.: Thermal transport from a heated moving surface. Trans. ASME J. Heat Transfer 108 (1986) 728-753

5. Vajravelu, K.; Mohapatra R. N.: On fluid dynamic drag reduction in some boundary layer flows. Acta Mech 81 (1990) 59-68

6. Pop, I.; Gorla, R. S. R.; Rashidi M.: The effect of variable viscosity on flow and heat transfer to a continuous moving flat plate. Int. J. Eng. Sci. 30 (1992)1-6

7. Ariman, T.; Turk, M. A.; Sylvester, N. D.: Microcontinuum fluid mechanics - a review. Int. J. Eng. Sci. 11 (1973) 905-930

8. Eringen, A. C.: Theory of micropolar fluids. J. Math. Mech. 16 (1966) 1-18

9. Eringen A. C.: Theory of thermomicrofluids. J. Math. Appl. 38 (1972) 480-496

10. Keller, H. B.; Cebeci, T.: Accurate numerical methods for boundary layers. I. Two dimensional laminar flows. In: Proc. 2nd Int. Conf. Numerical Methods in Fluid Dynamics. Lecture Notes in Physics, Vol. 8 New York: Springer 1971

11. Ahmadi, C.: Self-similar solution of incompressible micropolar boundary layer flow over a semiinfinite plate. Int. J. Eng. Sci. 14 (1976) 639-646

12. Arafa, A. A.; Gorla R. S. R.: Mixed convection boundary layer flow of a micropolar fluid along vertical cyclinders and needles. Int. J. Eng. Sci. 30 (1992) 1745-1751

13. Banks, W. H. H.: Similarity solutions of the boundary layer equations for a stretching wall. J. Mécan. Théor. Appl. 2 (1983) 375-392

14. Na, T. Y.: Numerical solution of natural convection flows past a non-isothermal vertical flat plate. Appl. Sci. Res. 33 (1978) 519-543

15. Na, T. Y.; Pop I.: Free convection flow past a vertical flat plate embedded in a saturated porous medium. Int. J. Eng. Sci. 21 (1983) 517-526

16. Ingham, D. B.; Pop, I.: Forced flow in a right-angled corner: higher order theory. Eur. J. Mech. 10 (1991) 313-331 